\title{
A Computational Study of Ultra-Wideband Versus Narrowband Microwave Hyperthermia for Breast Cancer Treatment
}

\author{
Mark Converse, Member, IEEE, Essex J. Bond, Member, IEEE, Barry D. Van Veen, Fellow, IEEE, and \\ Susan C. Hagness, Senior Member, IEEE
}

\begin{abstract}
We present a computational study comparing the performance of narrowband (NB) microwave hyperthermia for breast cancer treatment with a recently proposed ultra-wideband (UWB) approach. Space-time beamforming is used to preprocess input signals from both UWB and NB sources. The train of UWB pulses or the NB sinusoidal signals are then transmitted simultaneously from multiple antennas into the breast. Performance is evaluated using finite-difference time-domain electromagnetic (EM) and thermal simulations with realistic numerical breast phantoms derived from magnetic resonance images (MRIs) of the breast. We use three methods of mapping MRI data to complex permittivity data to account for uncertainty in the embodiment of the dielectric properties transitions in heterogeneous breast tissue. EM power-density deposition profiles and temperature profiles are compared for the UWB and NB cases in the three different breast phantoms. Dominant mechanisms that influence the efficacy of focusing UWB and NB signals in the breast are identified. The results of this study suggest that, while NB focusing performs reasonably well when the excitation frequency is optimized, UWB focusing consistently performs better, offering the potential for tighter focusing and greater reduction of hot spots, particularly in breast tissue, which exhibits distinct dielectric-properties boundaries within the tissue heterogeneity.
\end{abstract}

Index Terms-Breast cancer, electromagnetic (EM) hyperthermia, finite-difference time-domain (FDTD) method, microwave imaging, space-time beamforming, ultra-wideband (UWB) radar.

\section{INTRODUCTION}

$\mathbf{H}$ YPERTHERMIA is a well-known thermal therapy wherein the cytotoxic effects of elevated temperatures in tissue are induced to achieve cell death or render the cells more vulnerable to ionizing radiation and chemical toxins. Clinical studies have shown local hyperthermia to be effective in the treatment of a variety of cancers [1]-[4], including breast cancer [5]-[7], when delivered as an adjuvant to radiation and/or chemotherapy. The objective of hyperthermia treatment of cancer is to raise the temperature in the tumor volume above

Manuscript received August 2, 2005; revised December 22, 2005. This work was supported by the Department of Defense Breast Cancer Research Program under Award DAMD17-02-1-0625, by the National Science Foundation under Grant BES-0201880, and by the National Institutes of Health under Grant 1 F31 CA099933-01.

The authors are with the Department of Electrical and Computer Engineering, University of Wisconsin-Madison, Madison, WI 53706 USA (e-mail: converse@cae.wisc.edu; ejbond@wisc.edu; vanveen@engr.wisc.edu; hagness@engr.wisc.edu).

Digital Object Identifier 10.1109/TMTT.2006.872790
$42{ }^{\circ} \mathrm{C}-43{ }^{\circ} \mathrm{C}$ for a sufficient period of time while preserving normal physiological temperatures (well below $42{ }^{\circ} \mathrm{C}$ ) in the surrounding tissue. One of the persisting challenges in achieving this objective with noninvasive electromagnetic (EM) hyperthermia treatment is focusing EM power in the cancerous tissue while avoiding the introduction of auxiliary foci in normal tissue.

The use of an antenna array offers the opportunity for transmitting EM signals that constructively interfere at a desired location and destructively interfere elsewhere in space, thereby providing localized heating via selective absorption of EM energy. Numerous investigations have been conducted over the past several decades to explore and evaluate methods of focusing EM energy using arrays that transmit amplitude- and phase-adjusted narrowband (NB) signals [8], [9]. In contrast, until very recently, less attention has been given to the possibility of using multiple-frequency or ultra-wideband (UWB) signals. In 1998, Jacobsen proposed a multifrequency scheme based on the use of three NB signals distributed over a 520-MHz band and demonstrated that distributing the transmitted power over this frequency band produces fewer hot spots in the volume to be heated [10]. In 2004, we proposed and demonstrated the theoretical feasibility of an UWB microwave space-time beamforming system for focusing microwave energy at a lesion site in the breast [11]. In our UWB approach, an UWB pulse train is passed through a beamformer (a bank of time shifters and finite-impulse response (FIR) filters), which implements the frequency-dependent amplitude and phase adjustments in each channel to exploit coherent and incoherent combining of signals across frequency and space. Our preliminary results suggested that the necessary temperature gradients required for effective hyperthermia may be achieved with this technique.

Our previous feasibility study was motivated by the hypothesis that UWB focusing methods offer the potential for tighter focusing and a greater reduction of hot spots compared to NB methods. The rationale behind this hypothesis, as given in [11] from a frequency-domain perspective, is summarized here. The mainlobe for each frequency component of the transmitted UWB pulses attains a maximum at the focal location. Therefore, the total power at the focal location will be the coherent summation of power across frequency. The location and peak amplitudes of the sidelobes will be a function of array configuration, breast composition, and frequency. At positions away from the focal location, some frequencies will have sidelobe peaks while others will have nulls. Hence, for a fixed 
mainlobe power, the overall sidelobe levels are expected to be lower when transmitting UWB pulses rather than NB signals. While these arguments seem to be highly plausible, they were not verified by a formal study of UWB focusing compared with NB focusing in realistic breast tissue.

In this paper, we present a numerical investigation of the performance of UWB versus NB signals for focusing EM energy in the breast. Following the methodology in [11], we use an anatomically realistic finite-difference time-domain (FDTD) EM breast model, containing a 2-mm-diameter tumor, to simulate the absorbed EM power density distributions that result from the transmission of focused UWB or NB signals. An FDTD thermal model based on the Pennes bio-heat equation is used to simulate the temperature profiles which result from the simulated absorbed power density distributions. The results of this comparison study indicate that UWB focusing produces the desired elevated temperatures in the tumor region while preserving normal physiological temperatures throughout larger regions of normal breast tissue relative to NB focusing.

We have intentionally chosen to limit the comparison study to a relatively small tumor diameter of $2 \mathrm{~mm}$. As the tumor size decreases, the higher conductivity of malignant tissue makes less of a contribution toward selective microwave absorption, and the role that focusing plays becomes more critical. The small-tumor scenario therefore allows for a proper assessment of the validity of our hypothesis that tighter focusing and reduced hot-spots can be achieved with UWB focusing relative to NB focusing.

Section II describes the beamforming technique for focusing NB and UWB signals. Section III describes the numerical models and methods used to evaluate the efficacy of the UWB and NB hyperthermia techniques. In Section IV, we compare the two techniques by examining the absorbed EM power-density distributions and temperature profiles. These results are followed by concluding remarks in Section V.

\section{TRANSMIT BEAMFORMING}

A beamformer is a spatial filter that can be used with an array of antennas to focus energy at some desired location in a spatial field. NB beamformers are comprised of one complex weight in each antenna channel, while UWB beamformers contain a tapped delay line or FIR filter in each channel. The amplitude and phase in each channel is chosen to obtain constructive interference at the focus location and destructive interference elsewhere. The FIR filters in the UWB beamformer implement the amplitude and phase required for constructive/destructive interference as a function of frequency.

The goal of our transmit focusing design strategy is to maximize the energy deposited at a given location while minimizing energy deposited throughout the remainder of the breast region. We choose to place greater emphasis on minimizing energy deposition at interior regions than near the surface because a cooling medium can be used to prevent unhealthy temperatures near the skin. A time-domain approach is used for the UWB beamformer design to jointly optimize the design criterion across all frequencies in the band of interest. In contrast, solving a series of decoupled design problems, one for each frequency as in [11], results in a suboptimal solution, since it does not take into account the fact that the net energy responsible for heating is the integral of the energy at each frequency. Independently optimizing the energy at each frequency is not equivalent to optimizing the integral.

Assume that an array of $N$ antennas is located in a coupling/ cooling medium surrounding the breast. We represent the analytical model of the frequency response associated with propagation through the coupling medium and normal breast tissue from the $n$th antenna to location $\mathbf{r}_{0}$ by $T_{n}\left(\omega, \mathbf{r}_{0}\right)$. The analytical propagation model employed here is identical to that described in [11]. The FIR filter in the $n$th channel has coefficients represented by the $L \times 1$ vector $\mathbf{h}_{n}=\left[h_{n 0}, h_{n 1}, \cdots, h_{n(L-1)}\right]^{T}$, where superscript $T$ denotes the vector/matrix transpose. The filter length $L$ is chosen empirically to balance performance and complexity. The frequency response of the $n$th filter is written as

$$
H_{n}(\omega)=\sum_{\ell=0}^{L-1} h_{n \ell} e^{-j \omega \ell T_{s}}=\mathbf{h}_{n}^{T} \mathbf{d}(\omega)
$$

where $\mathbf{d}(\omega)=\left[1, e^{-j \omega T_{s}}, \cdots, e^{-j \omega(L-1) T_{s}}\right]^{T}$ and $T_{s}$ is the sampling interval. The total weighted energy deposited in a region $\Phi$ of the breast in the UWB case is thus obtained as

$$
\begin{aligned}
E_{\Phi} & =\int_{\Phi} \int_{\Omega} W(\mathbf{r})\left|\sum_{n=1}^{N} H_{n}(\omega) T_{n}(\omega, \mathbf{r})\right|^{2} d \omega d \mathbf{r} \\
& =\int_{\Phi} \int_{\Omega} W(\mathbf{r})\left|\sum_{n=1}^{N} \mathbf{h}_{n}^{T} \mathbf{d}(\omega) T_{n}(\omega, \mathbf{r})\right|^{2} d \omega d \mathbf{r}
\end{aligned}
$$

where $\Omega$ represents the temporal frequency band of interest. We choose the weighting term $W(\mathbf{r})$ in (2) as $W(\mathbf{r})=e^{-c\left|\mathbf{r}-\mathbf{r}_{0}\right|}$ in order to emphasize the region near $\mathbf{r}_{0}$ while de-emphasizing those positions distant from $\mathbf{r}_{0}$. Usually, $\mathbf{r}_{0}$ is chosen near the center of the breast to de-emphasize energy deposition near the skin. The constant $c$ controls the degree of de-emphasis with distance from $\mathbf{r}_{0}$.

Let the $N L \times 1$ vector $\mathbf{h}=\left[\mathbf{h}_{1}^{T}, \mathbf{h}_{2}^{T}, \cdots, \mathbf{h}_{N}^{T}\right]^{T}$ be the concatenation of the weight vectors from each channel. Define $\mathbf{d}_{n}(\omega, \mathbf{r})=T_{n}(\omega, \mathbf{r}) \mathbf{d}(\omega), 1 \leq n \leq N$, and let $\mathbf{d}(\omega, \mathbf{r})=\left[\mathbf{d}_{1}^{T}(\omega, \mathbf{r}), \mathbf{d}_{2}^{T}(\omega, \mathbf{r}), \cdots, \mathbf{d}_{N}^{T}(\omega, \mathbf{r})\right]^{T}$. We may now rewrite (2) as

$$
\begin{aligned}
E_{\Phi} & =\int_{\Phi} \int_{\Omega} W(\mathbf{r})\left|\mathbf{h}^{T} \mathbf{d}(\omega, \mathbf{r})\right|^{2} d \omega d \mathbf{r} \\
& =\mathbf{h}^{T} \int_{\Phi} \int_{\Omega} W(\mathbf{r}) \mathbf{d}(\omega, \mathbf{r}) \mathbf{d}^{H}(\omega, \mathbf{r}) d \omega d \mathbf{r h} \\
& =\mathbf{h}^{T} \mathbf{Q h}
\end{aligned}
$$

where $\mathbf{Q}=\int_{\Phi} \int_{\Omega} W(\mathbf{r}) \mathbf{d}(\omega, \mathbf{r}) \mathbf{d}^{H}(\omega, \mathbf{r}) d \omega d \mathbf{r}$ and superscript $H$ denotes complex-conjugate transpose. The energy deposited at the focus location $\mathbf{r}_{f}$ in the UWB case is $E_{f}=\mathbf{h}^{T} \mathbf{Q}_{f} \mathbf{h}$, 
where $\mathbf{Q}_{f}=\int_{\Omega} \mathbf{d}\left(\omega, \mathbf{r}_{f}\right) \mathbf{d}^{H}\left(\omega, \mathbf{r}_{f}\right) d \omega$. Our transmit focusing design goal is now concisely expressed as

$$
\mathbf{h}=\arg \max _{\mathbf{h}} \frac{\mathbf{h}^{T} \mathbf{Q}_{f} \mathbf{h}}{\mathbf{h}^{T} \mathbf{Q h}+\xi \mathbf{h}^{T} \mathbf{h}} .
$$

The term $\xi \mathbf{h}^{T} \mathbf{h}$ in (4) is used to penalize solutions with a large norm, since such solutions are not robust to modeling errors and other slight perturbations [12]. We choose $\xi$ to be $\|\mathbf{Q}\| / 10$, which weights the norm of $\mathbf{h}$ at approximately $10 \%$ of the total energy $E_{\Phi}$ in the optimization criterion.

Carrying out the maximization in (4) requires solving the generalized eigenvalue problem

$$
\mathbf{Q}_{f} \mathbf{h}=\lambda\left(\mathbf{Q}+\xi \mathbf{I}_{N L}\right) \mathbf{h}
$$

where $\lambda$ is the eigenvalue and $\mathbf{I}_{N L}$ is the $N L \times N L$ identity matrix. Thus, the solution to (4) is the eigenvector corresponding to the largest eigenvalue of $\left(\mathbf{Q}+\xi \mathbf{I}_{N L}\right)^{-1} \mathbf{Q}_{f}$.

The NB case is obtained by reducing the filter length in each channel to $L=1$. The frequency of interest is denoted by $\omega_{0}$. The optimization problem (4) is applicable with $\mathbf{Q}=\int_{\Phi} W(\mathbf{r}) \mathbf{d}\left(\omega_{0}, \mathbf{r}\right) \mathbf{d}^{H}\left(\omega_{0}, \mathbf{r}\right) d \mathbf{r}$ and $\mathbf{Q}_{f}=\mathbf{d}\left(\omega_{0}, \mathbf{r}_{f}\right) \mathbf{d}^{H}\left(\omega_{0}, \mathbf{r}_{f}\right)$. The solution to (4) for this case is expressed in closed form as

$$
\mathbf{h}_{n b}=\alpha\left(\mathbf{Q}+\xi \mathbf{I}_{N L}\right)^{-1} \mathbf{d}\left(\omega_{0}, \mathbf{r}_{f}\right)
$$

where $\alpha$ is any real scalar. We choose $\alpha=\left(\mathbf{d}^{H}\left(\omega_{0}, \mathbf{r}_{f}\right)(\mathbf{Q}+\right.$ $\left.\left.\xi \mathbf{I}_{N L}\right)^{-1} \mathbf{d}\left(\omega_{0}, \mathbf{r}_{f}\right)\right)^{-1}$.

The beamformer that is used to generate the results in this paper assumes that the number of antennas is $N=17$. The filter length in each channel is $L=21$ for the UWB case and $L=1$ for the NB case. The sampling period is $20 \mathrm{ps}$. The frequency band of interest is $\Omega=[-11,-1] \cup[1,11] \mathrm{GHz}$ and $\Phi$ is the breast interior. The weighting factor $c$ is chosen to be 0.9 in units of $\mathrm{cm}^{-1}$.

\section{Numerical Models for Performance Evaluation}

We examine the differences between UWB and NB focusing for hyperthermia treatment by performing two-dimensional (2-D) simulations to calculate distributions of absorbed power density and temperature profiles throughout the breast. This approach allows for efficient evaluation of the relative merits of these two techniques and simplifies the problem by eliminating configurational complications and polarization concerns. While we expect some quantitative differences to be observed between 2-D and three-dimensional (3-D) focusing, the qualitative conclusions drawn from the comparison of UWB and NB focusing in two dimensions should be valid and extendable to three dimensions. Both the 2-D EM and thermal models are similar to the magnetic resonance image (MRI)-derived breast models presented in [11] except for the methods used to map MRI data to complex permittivity data and the thermal parameters used in the breast models. The key features of these models are summarized below.

\section{A. Anatomically Based Numerical Breast Phantoms}

The configuration used in this study mimics that of a patient lying in the prone (or face-down) position with the breast

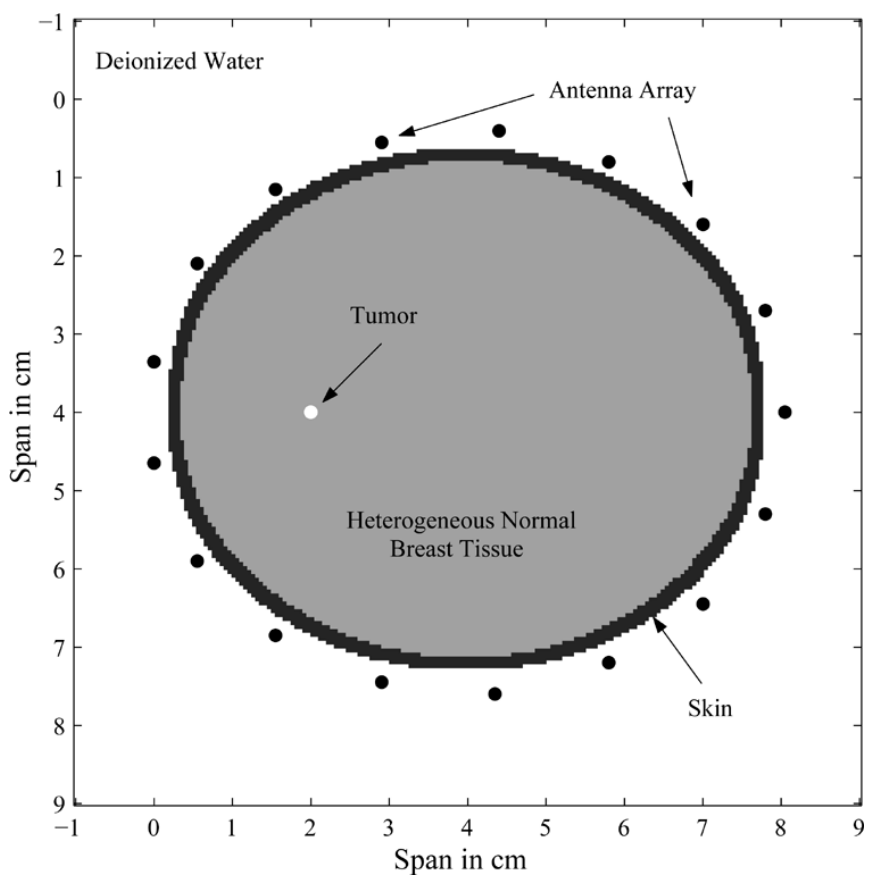

Fig. 1. 2-D FDTD model of the hyperthermia treatment configuration for a patient lying in the prone position. The MRI-derived breast model contains a 2 -mm-diameter malignant lesion shown by a white dot. The 17 black dots near the surface of the breast represent antenna locations. The realistic nature of the heterogeneous normal tissue is illustrated in Fig. 2.

extending through an opening in the treatment table. In this position, the antenna array encircles the pendulous breast allowing for easy access to the full volume of the breast. The prone configuration is represented in two dimensions by a coronal plane through the breast with antennas surrounding the breast, as shown in Fig. 1. A 2-mm-diameter malignant tumor is inserted into the breast model at a distance of $1.5-2.0 \mathrm{~cm}$ from the surface of the breast. Each phantom is composed of four different media types: heterogeneous normal breast tissue, skin, malignant tumor, and deionized water (the coupling/cooling medium).

\section{B. EM Model}

A 2-D $\mathrm{TM}_{z}$ FDTD-based EM model is used to calculate the absorbed power density distributions that arise from the focusing of UWB and NB microwave signals in the breast. The FDTD EM model solves Maxwell's equations on a discrete spatial grid comprised of a numerical breast phantom and antenna array configuration shown in Fig. 1. The grid resolution used for these simulations is $0.5 \times 0.5 \mathrm{~mm}$. The antennas are modeled as electric-current sources that radiate the set of NB or UWB signals designed using the procedure described in Section II.

The dispersive nature of the media properties is incorporated into the FDTD model using an auxiliary differential equation technique [13]. The dispersion characteristics are treated using single-pole Debye dispersion expressions of the following form:

$$
\epsilon_{r}^{*}(\omega)=\epsilon_{r}^{\prime}(\omega)-j \epsilon_{r}^{\prime \prime}(\omega)=\epsilon_{\infty}+\frac{\epsilon_{s}-\epsilon_{\infty}}{1+j \omega \tau}-j \frac{\sigma_{s}}{\omega \epsilon_{\circ}} .
$$

Here, $\epsilon_{\infty}$ is the relative permittivity at infinite frequency, $\epsilon_{s}$ is the static relative permittivity, $\sigma_{s}$ is the static conductivity, and $\tau$ is the relaxation time constant. Table I lists the specific Debye 
TABLE I

Debye Parameters for the DisPersive Materials InCluded IN THE EM Model AND the Resulting Dielectric Properties AT 6 GHz

\begin{tabular}{|c||c|c|c|c|c|c|}
\hline \multicolumn{1}{|c||}{ Media } & \multicolumn{6}{c|}{ Electromagnetic Media Characteristics } \\
\cline { 2 - 7 } & $\epsilon_{\infty}$ & $\epsilon_{s}$ & $\sigma_{s}$ & $\tau$ & $\epsilon_{r}(6 \mathrm{GHz})$ & $\sigma(6 \mathrm{GHz})$ \\
\hline \hline DI water & 4.55 & 77.11 & 0.0002 & $7.37 \mathrm{e}-12$ & 71.91 & 6.25 \\
\hline skin & 4.00 & 37.00 & 1.10 & $7.23 \mathrm{e}-12$ & 34.72 & 3.89 \\
\hline tumor & 3.99 & 54.00 & 0.70 & $7.0 \mathrm{e}-12$ & 50.74 & 4.82 \\
\hline fatty tissue & 7.00 & 10.00 & 0.15 & $7.0 \mathrm{e}-12$ & 9.80 & 0.40 \\
\hline average tissue & 6.57 & 16.29 & 0.23 & $7.0 \mathrm{e}-12$ & 15.66 & 1.03 \\
\hline fibroglandular tissue & 6.14 & 21.57 & 0.31 & $7.0 \mathrm{e}-12$ & 21.5 & 1.7 \\
\hline
\end{tabular}

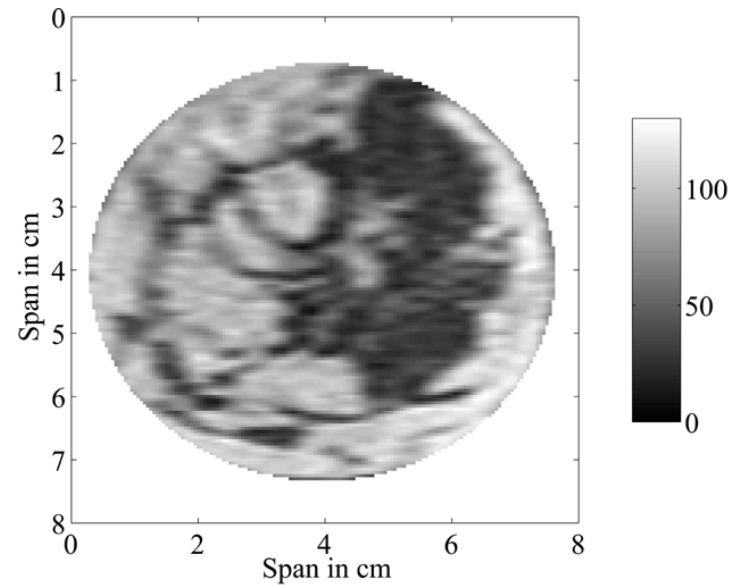

(a)

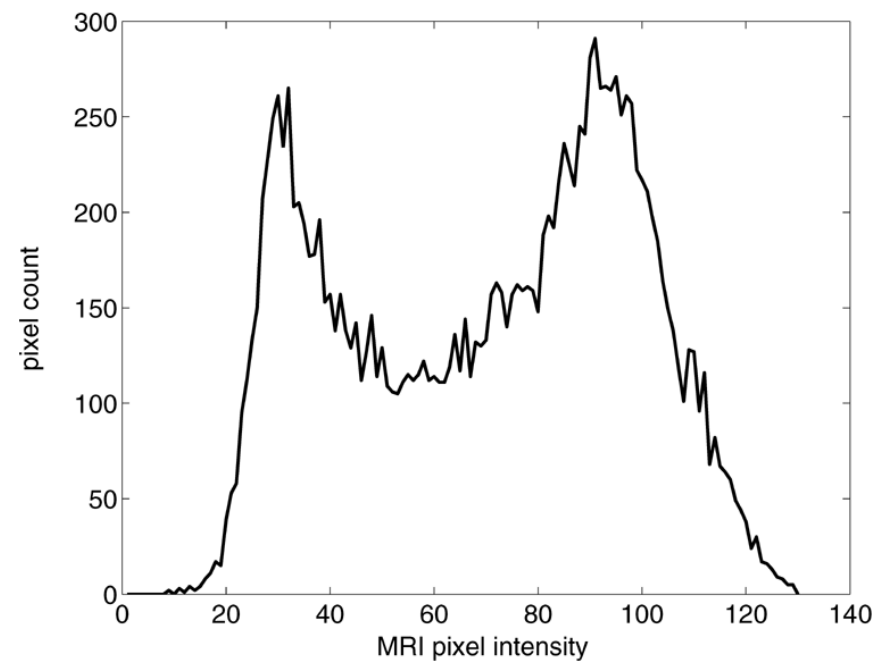

(b)

Fig. 2. MRI data from which the FDTD breast model is derived. (a) MRI image showing pixel intensity as a function of position within the coronal plane of the breast. (b) Histogram of MRI pixel intensities.

parameters for each material [14]-[16]. Note that $\epsilon_{r}(6 \mathrm{GHz})$ and $\sigma(6 \mathrm{GHz})$ denote the values of dielectric constant and conductivity generated by the Debye model at $6 \mathrm{GHz}$ - which is the center of the frequency band of interest.

The anatomically realistic variation of the frequency-dependent permittivity and conductivity in the interior of the breast is derived from the density variation within a high-resolution breast MRI data set. The original MRI data are shown as an image in Fig. 2(a) and as a histogram depicting the range and distribution of pixel intensities in Fig. 2(b). Darker regions (low pixel intensities) represent denser fibroglandular tissue while the lighter regions (high pixel intensities) indicate less dense adipose tissue. In creating the MRI-derived FDTD EM model, we do not attempt to directly estimate the complex permittivity from the MRI pixel intensity data; rather, we map the MRI data to representative values of complex permittivity using the MRI data as a template of heterogeneity.

The choice of complex permittivity values is not a definitive one because of the uncertainty that exists in the literature on the dielectric properties of normal breast tissue at microwave frequencies [17]. Three dielectric spectroscopy studies [18]-[20] suggest that normal breast tissue is a low-dielectric-constant, low-loss material and that the within-patient variability in dielectric properties is less than $\pm 10 \%$ around the nominal values. However, the nominal values across these three studies are not in agreement. Other studies [21]-[23] suggest that the impact of normal breast tissue heterogeneity on dielectric-property variability is more significant, because the dielectric properties of fat and fibroglandular tissue are distinctly different. Tissue heterogeneity in the breast may, in fact, explain the discrepancies observed across the different studies of [18]-[20].

We account for this dielectric-property uncertainty in our investigation by creating three different types of MRI-derived breast models for use in the UWB versus NB performance comparison. These models differ in the manner in which the MRI pixel intensities in the heterogeneous breast interior are mapped to Debye parameters $\left(\epsilon_{\infty}, \epsilon_{s}\right.$, and $\left.\sigma_{s}\right)$. The three mapping methods-uniform, piecewise linear, and bimodal-are described below. In all three cases, the Debye relaxation time $(\tau)$ is treated as a constant throughout the breast interior.

1) Uniform Mapping: This method follows the strategy presented in [11] and [24] and uses a smooth linear mapping between the range of MRI pixel intensities in the breast interior and a range of Debye parameters around a median baseline. The row labeled as "average tissue" in Table I summarizes the Debye parameters for the median baseline and the corresponding dielectric constant and conductivity at $6 \mathrm{GHz}$. The histogram of Fig. 3(a) shows the range and distribution of dielectric constants at $6 \mathrm{GHz}$ that result from applying this uniform mapping scheme to the data of Fig. 2(b) assuming a variability of $\pm 50 \%$ about the median. This result illustrates a general feature of all three mapping schemes - that low MRI pixel intensities (dense fibroglandular tissue) are assigned Debye parameters yielding the largest values of dielectric constant and conductivity while high MRI pixel intensities (fatty tissue) are assigned the smallest values.

2) Piecewise-Linear Mapping: This method assumes fatty and fibroglandular tissue are distinct tissue types, each with its own set of median Debye parameters and degree of variability about the median. Visual inspection of the MRI image is used to 


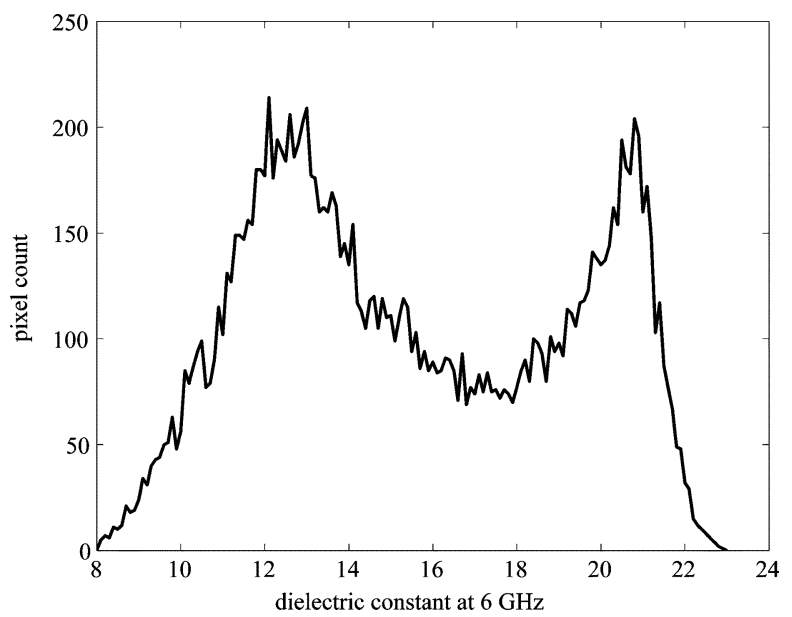

(a)

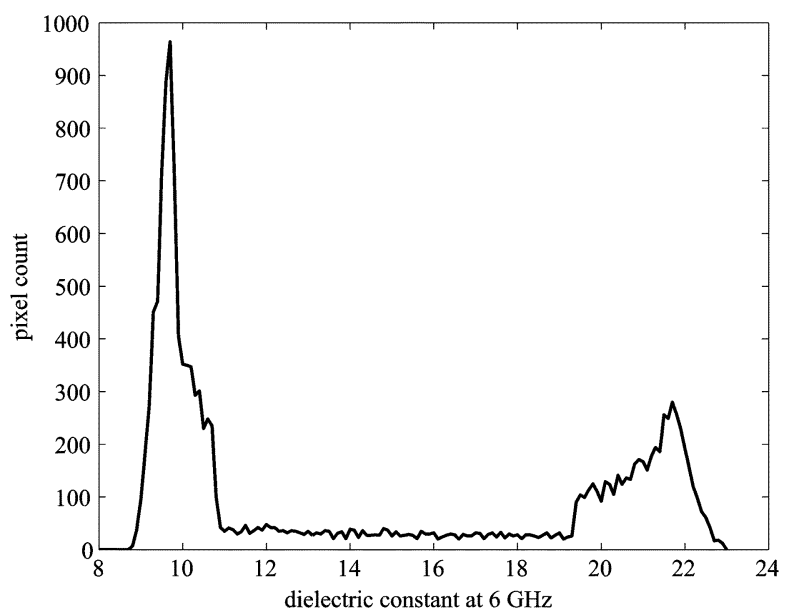

(b)

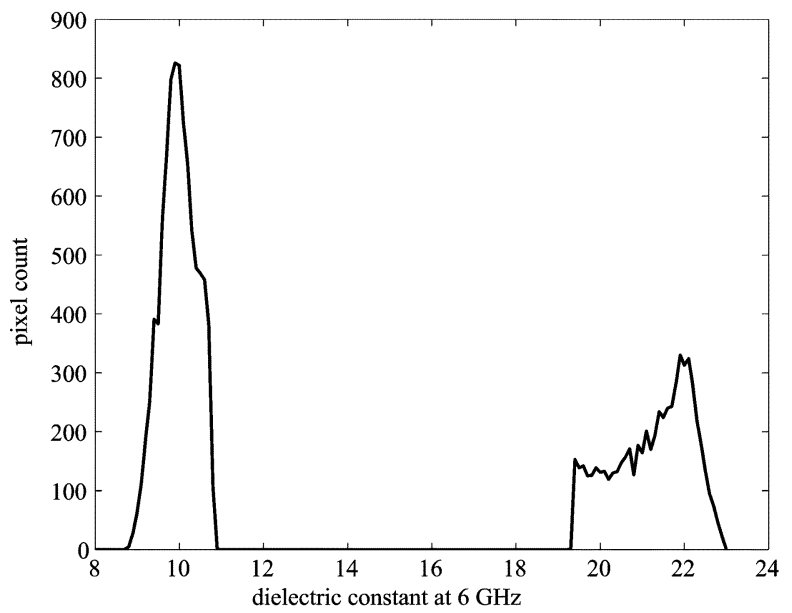

(c)

Fig. 3. Histograms of the dielectric constant at $6 \mathrm{GHz}$ resulting from: (a) uniform, (b) piecewise-linear, and (c) bimodal mapping schemes applied to the MRI pixel intensities in Fig. 2.

determine intensity thresholds for the fatty, fibroglandular, and transition regions in the histogram of Fig. 2(b). The intensity at which the local peak occurs within the higher end of the intensity spectrum of Fig. 2(b) is mapped to the median Debye parameters chosen for fatty tissue. The maximum pixel intensity within this fatty region of the histogram is mapped to the
TABLE II

THERMAL CONSTANTS USED IN THE NUMERICAL MODEL OF THE Bio-Heat Equation Derived From Data IN [26]-[43]

\begin{tabular}{|c||c|c|c|c|c|}
\hline \multicolumn{1}{|c||}{ Media } & \multicolumn{5}{c|}{ Thermal Media Characteristics } \\
\cline { 2 - 6 } & $K\left[\frac{\mathrm{W}}{\mathrm{m} \cdot{ }^{\circ} \mathrm{C}}\right]$ & $C_{p}\left[\frac{\mathrm{J}}{\mathrm{kg} \cdot{ }^{\circ} \mathrm{C}}\right]$ & $\rho\left[\frac{\mathrm{kg}}{\mathrm{m}^{3}}\right]$ & $A_{0}\left[\frac{\mathrm{W}}{\mathrm{m}^{3}}\right]$ & $B\left[\frac{\mathrm{W}}{{ }^{\circ} \mathrm{Cm}^{3}}\right]$ \\
\hline \hline DI water & 0.6 & 4186 & 1000 & 0 & 0 \\
\hline skin & 0.397 & 3765 & 1085 & 1620 & 5929 \\
\hline tumor & 0.496 & 3049 & 1182 & 5500 & 5350 \\
\hline breast & 0.306 & 2279 & 1069 & 350 & 2229 \\
\hline
\end{tabular}

minimum fatty tissue Debye parameters, and the minimum fatty tissue pixel intensity is mapped to the maximum fatty tissue Debye parameters. The same process is applied to the fibroglandular tissue region clustered at the lower end of the pixel intensity spectrum. Pixel intensities within the transition region are mapped to Debye parameters that span the range between the fatty maximum and fibroglandular minimum. Fig. 3(b) shows a histogram of the resulting dielectric constant at $6 \mathrm{GHz}$ when the median Debye parameters are assigned the values listed in Table I for fatty and fibroglandular tissue and the variation about each of the two medians is chosen to be $\pm 10 \%$.

3) Bimodal Mapping: This method also assumes two distinct tissue types in the interior of the breast, but does not allow for a transition region. A single-intensity threshold is used to set the boundary between the fatty and fibroglandular tissues. The pixel intensities within the fat and fibroglandular regions are mapped to Debye parameters in a manner similar to that described above for the piecewise-linear mapping method. Fig. 3(c) shows a histogram of the resulting dielectric constant at $6 \mathrm{GHz}$ when the median Debye parameters are assigned the values listed in Table I for fatty and fibroglandular tissue and the variation about each of the two medians is chosen to be $\pm 10 \%$. Comparing Fig. 3(c) with (3b), we conclude that the bimodal mapping scenario introduces the sharpest contrasts and, therefore, the greatest scattering within the propagation medium because of the jump discontinuities that exist in the dielectric properties in the absence of a transition region.

We note that these three methods yield FDTD models of the breast with slightly different average dielectric properties of the breast interior. For example, at $6 \mathrm{GHz}$, the spatially averaged dielectric constant and conductivity values are as follows: $\epsilon_{r}=15.5$ and $\sigma=0.99 \mathrm{~S} / \mathrm{m}$ for the uniformly mapped model, $\epsilon_{r}=14.3$ and $\sigma=0.91 \mathrm{~S} / \mathrm{m}$ for the piecewise linearly mapped model, and $\epsilon_{r}=14.61$ and $\sigma=0.93 \mathrm{~S} / \mathrm{m}$ for the bimodally mapped model. The analytical propagation model used in the design of the beamformer (discussed in Section II) requires an assumed average for the dielectric properties of the interior of the breast. We generated the results reported in this paper (in Section IV) using beamformers that were designed with average values corresponding exactly to the specific breast phantom under consideration. We have verified that there is no discernable change in the performance when beamformers designed using one of the other sets of average values are employed in the focusing of the microwave signals. In fact, the beamformer performance is quite robust with respect to much larger mismatches between the average dielectric properties assumed in the beamformer design and the actual average dielectric properties of the interior breast tissue environment. 


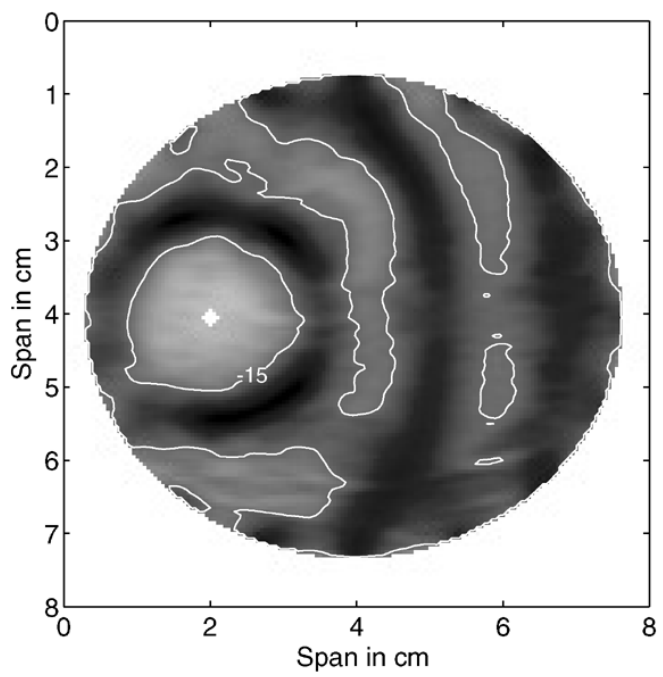

(a)

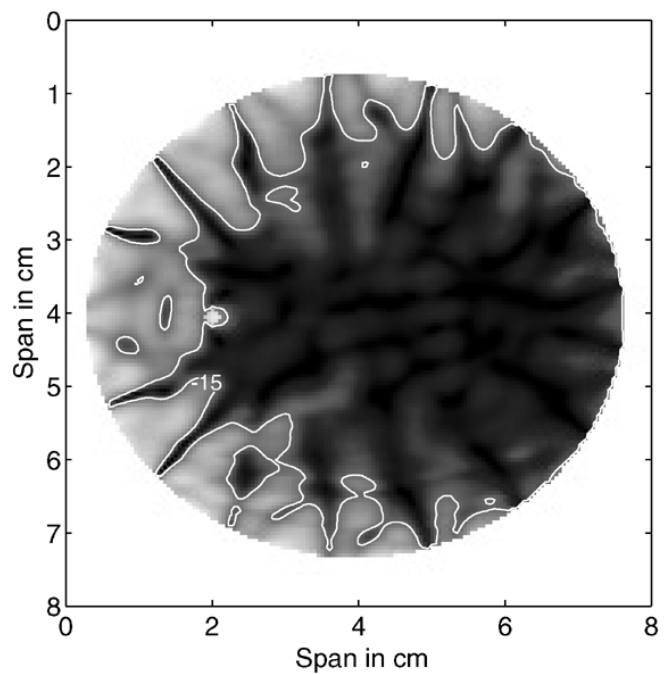

(c)
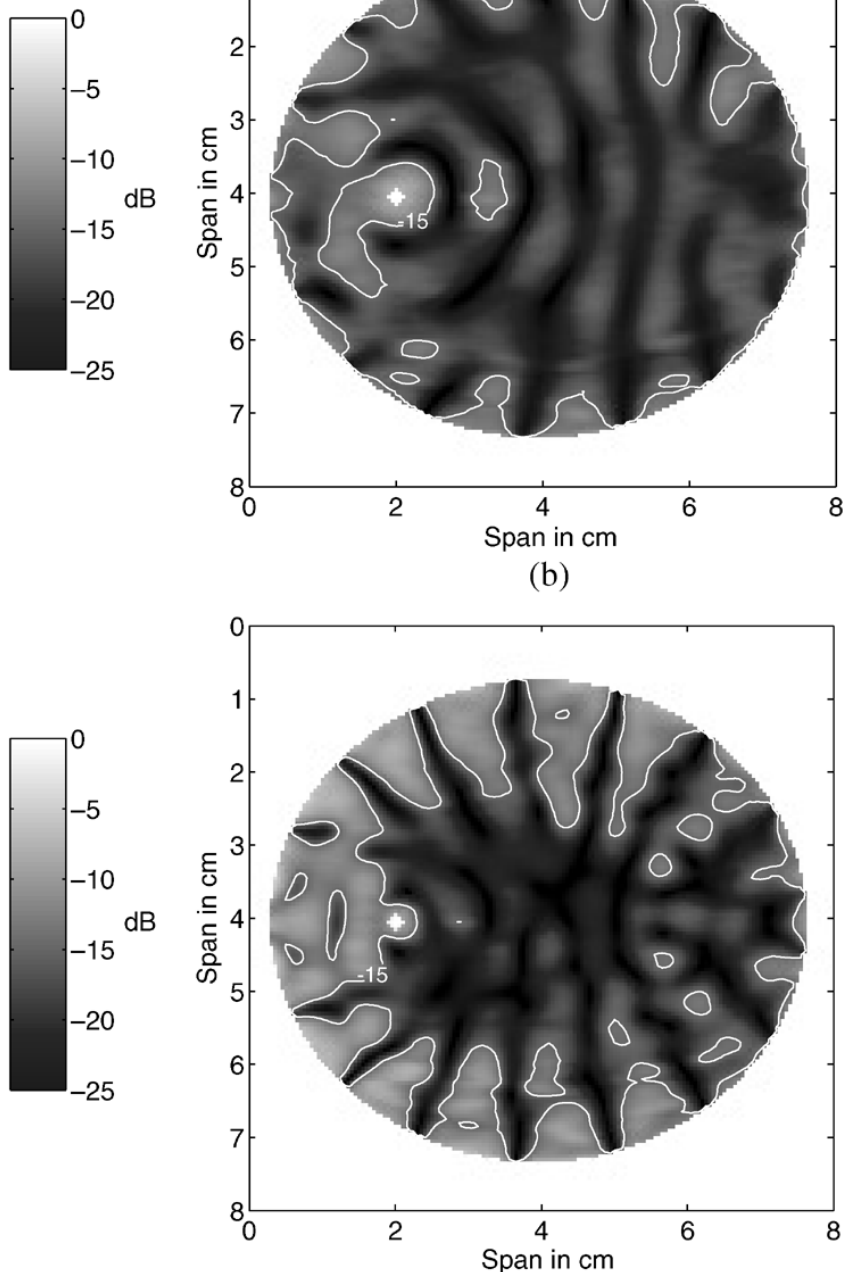

(d)

(b)

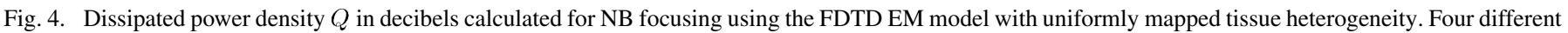

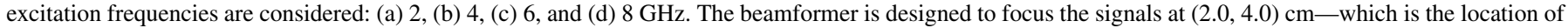
a 2-mm-diameter tumor.

EM power deposition is a well-accepted figure of merit for evaluating the effectiveness of the focusing strategy employed in hyperthermia. The heating potential $(Q)$, that is, the power dissipated per unit volume, is calculated as a function of location in the breast in one of two ways. For the UWB simulations, we calculate $Q$ using the FDTD-computed time-domain field quantities as follows: [25]:

$$
Q_{i, j}=R \sum_{n=0}^{n_{\max }}\left(\sigma_{s_{i, j}}\left(E_{z_{i, j}}^{n}\right)^{2}+E_{z_{i, j}}^{n} \frac{\partial D_{z_{i, j}}^{n}}{\partial t}\right) \Delta t \quad\left[\frac{W}{m^{3}}\right]
$$

where $i$ and $j$ are the computational lattice indices and $R$ is the assumed pulse repetition rate. For the NB (single-frequency) simulations, we calculate $Q$ using the FDTD-derived phasor field quantities as follows:

$$
Q_{i, j}(\omega)=0.5 \sigma_{\mathrm{eff}_{i, j}}(\omega)\left|\tilde{E}_{z_{i, j}}(\omega)\right|^{2} \quad\left[\frac{W}{m^{3}}\right]
$$
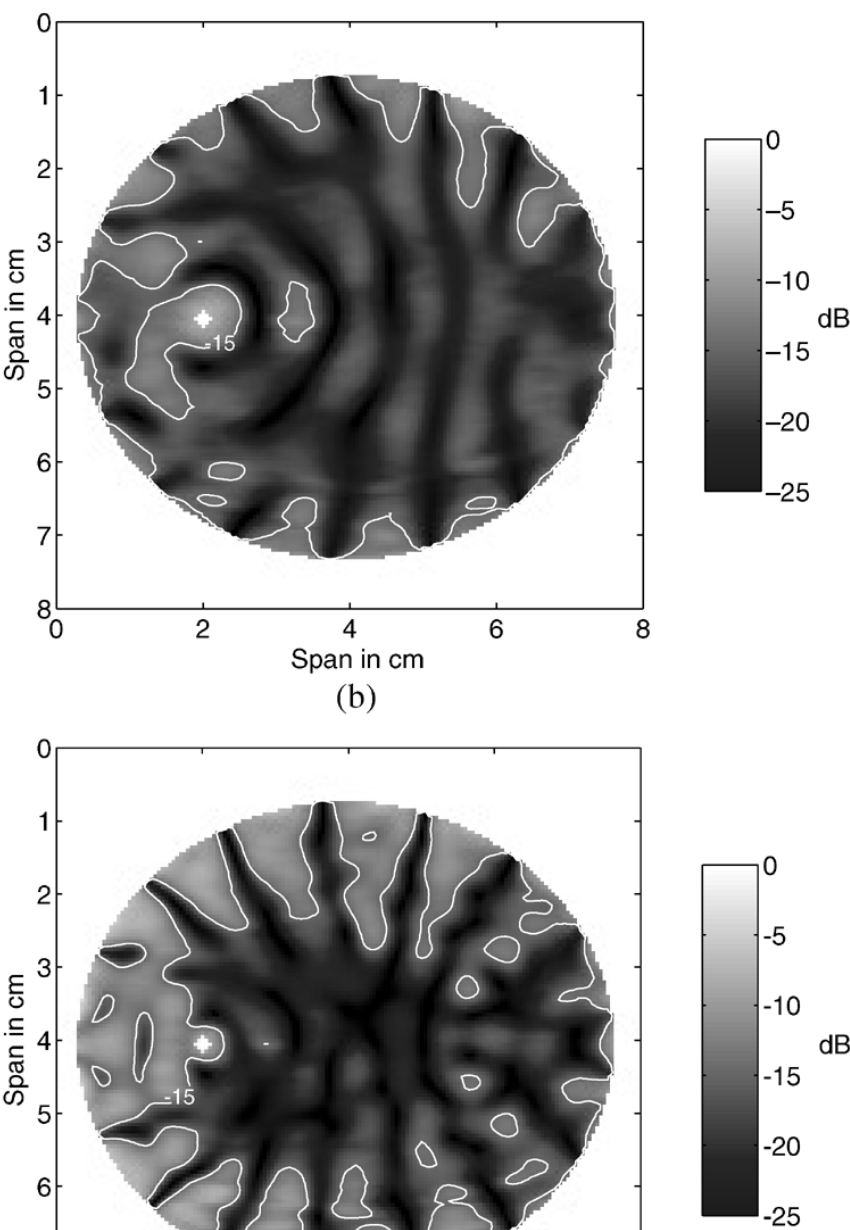

where $\omega$ is the frequency of the transmitted NB signals, $\sigma_{\text {eff }}(\omega)=\omega \epsilon_{0} \epsilon_{r}^{\prime \prime}(\omega)$, and $\tilde{E}_{z}(\omega)$ is the electric field phasor.

\section{Thermal Model}

We have also constructed a 2-D FDTD thermal model to compare UWB and NB focusing on the basis of temperature profiles. The model is based on the well known bio-heat equation:

$$
\begin{aligned}
C_{p}(\mathbf{r}) \rho(\mathbf{r}) \frac{\partial T(\mathbf{r})}{\partial t}=\nabla \cdot & (K(\mathbf{r}) \nabla T(\mathbf{r}))+A_{0}(\mathbf{r})+Q(\mathbf{r}) \\
& -B(\mathbf{r})\left(T(\mathbf{r})-T_{B}\right) \quad\left[\frac{W}{m^{3}}\right]
\end{aligned}
$$

which is discretized using the method of [26]. Here, $C_{p}$ is the specific heat, $\rho$ is the density, $K$ is the thermal conductivity, $A_{0}$ represents metabolic heat production, $Q$ is the heating potential computed in the FDTD EM simulation, $B$ is a constant representing the heat exchange mechanism due to capillary blood perfusion, and $T_{B}$ is the blood temperature (assumed to be at body temperature). A discussion of the thermal parameters and their 


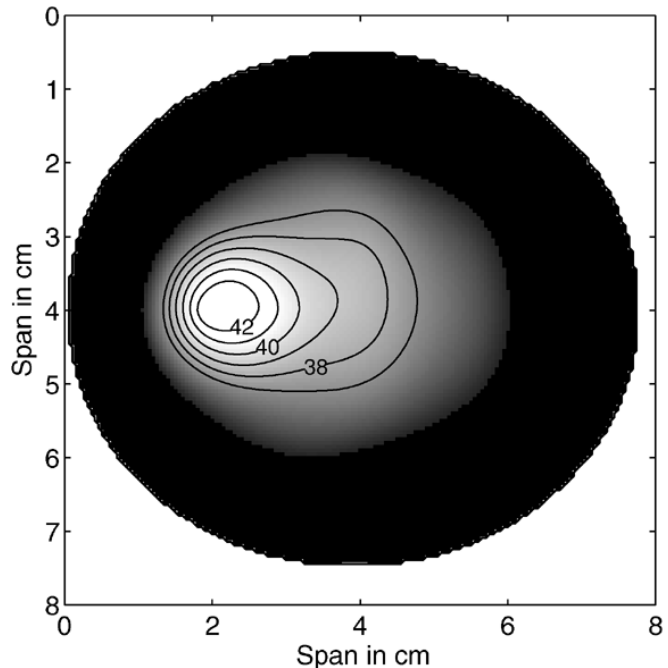

(a)

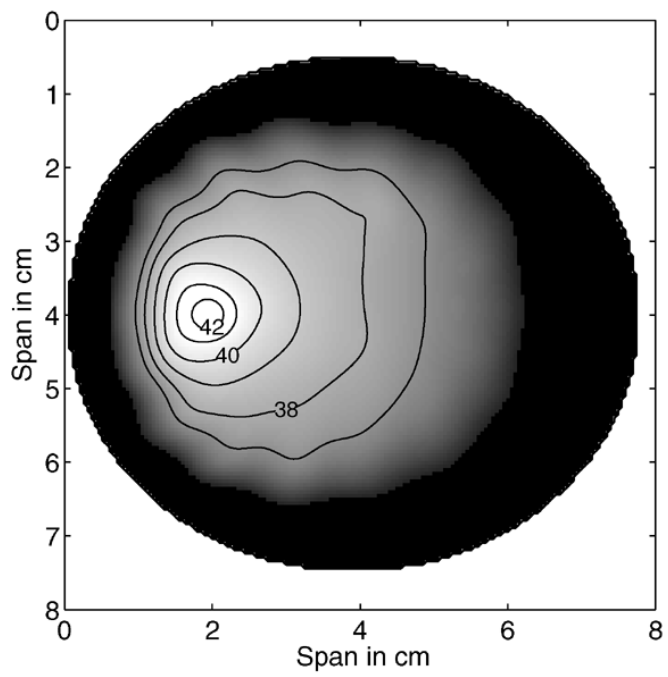

(c)
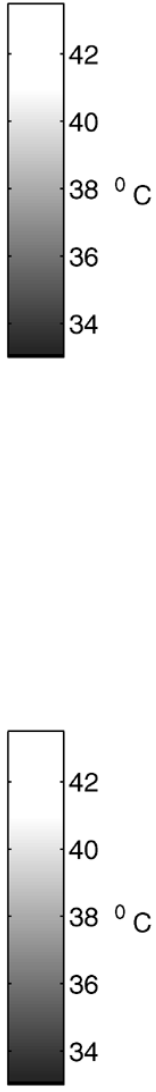

Fig. 5. Temperature distribution calculated using the FDTD thermal model with the power deposition pattern of: (a) Fig. 4(a) (2 GHz), (b) Fig. 4(b) (4 GHz), (c) Fig. 4(c) $(6 \mathrm{GHz})$, and (d) Fig. 4(d) $(8 \mathrm{GHz})$. role in the bio-heat equation is found in [26]. The thermal model consists of the same four media as the EM model and uses the same computational lattice. Thermal properties for the different media, listed in Table II, were obtained by averaging numerous values found in the literature [27]-[43]. Note that, while the heterogeneity of normal breast tissue is reflected in the dielectric properties of the FDTD EM model, it is not reflected in the properties of the FDTD thermal model, i.e., the thermal properties are assumed to be constant throughout the normal breast tissue region of the thermal model. Convective boundary conditions with a convective coefficient of $300 \mathrm{~W} / \mathrm{m}^{2} \cdot \mathrm{K}$ are used in this model to simulate the effects of chilled $\left(15^{\circ} \mathrm{C}\right)$ water at the skin surface. The value chosen for the convective coefficient is based upon an extrapolation of the experimental results for a cooling system used in ultrasound vasectomy [44].

The thermal simulation proceeds as follows. First, as an initialization step, an equilibrium temperature distribution is determined for the breast in air at room temperature with no external sources. Then, we assume that the breast is immersed in

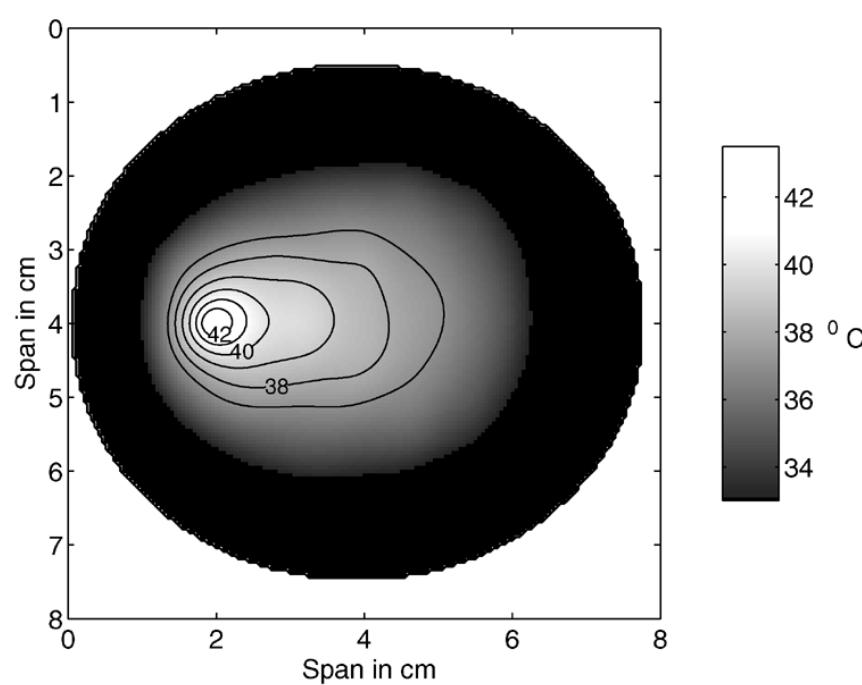

(b)

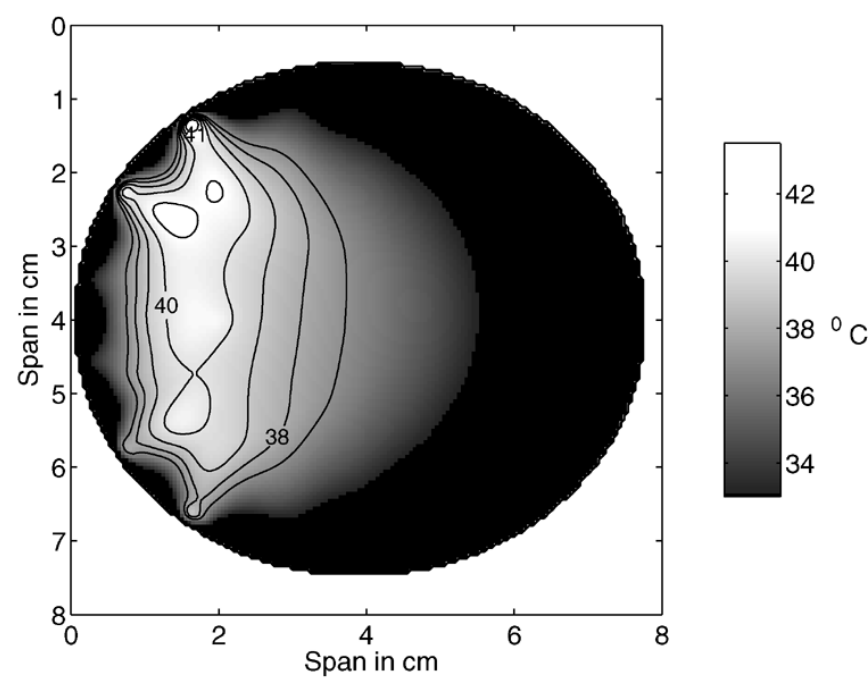

(d) the coupling/cooling medium for two minutes and simulate the resulting temperature distribution. Finally, the spatial distribution of $Q$, as computed by the FDTD EM simulation, is introduced throughout the breast, and the simulation is run until a steady-state condition is reached.

\section{Simulation Results AND Discussion}

Here, we compare the simulation results for UWB and NB beamforming in the numerical breast phantoms described in Section III. Each breast phantom consists of one of the three FDTD EM models (e.g., uniformly, piecewise linearly, or bimodally mapped tissue heterogeneity) coupled with the sole FDTD thermal model. In all figures showing the spatial distribution of $Q$, the skin region is excluded from view. Water cooling is sufficient to minimize skin heating, as is evident in all temperature-profile figures where the skin region is included.

First, we consider the effectiveness of NB hyperthermia for the numerical phantom comprised of the FDTD breast model with uniformly mapped tissue heterogeneity and a tumor centered at 


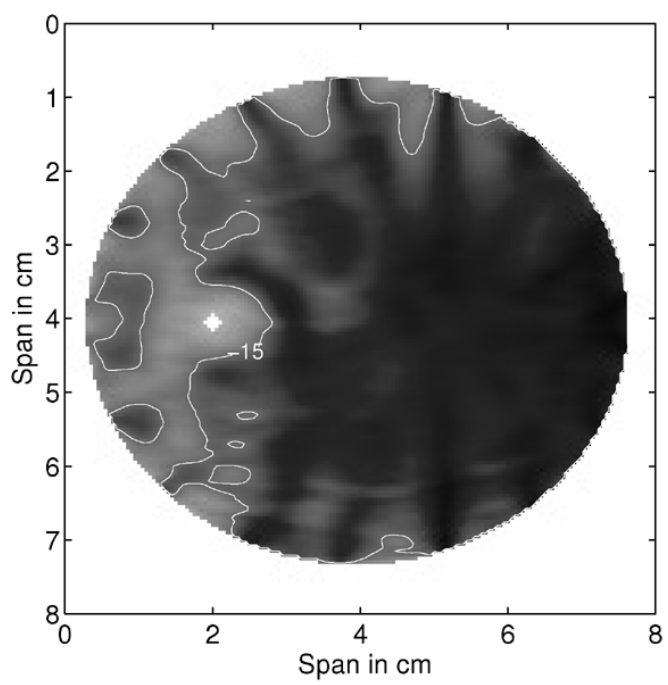

(a)

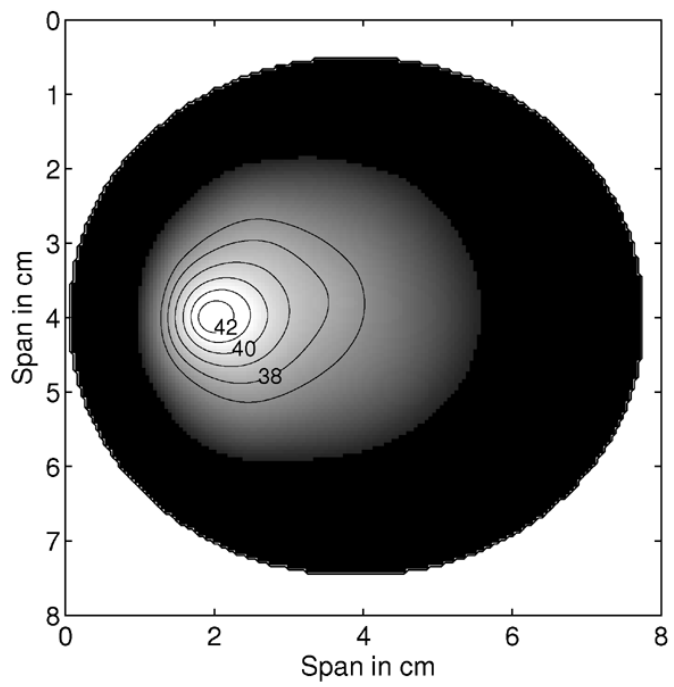

(c)

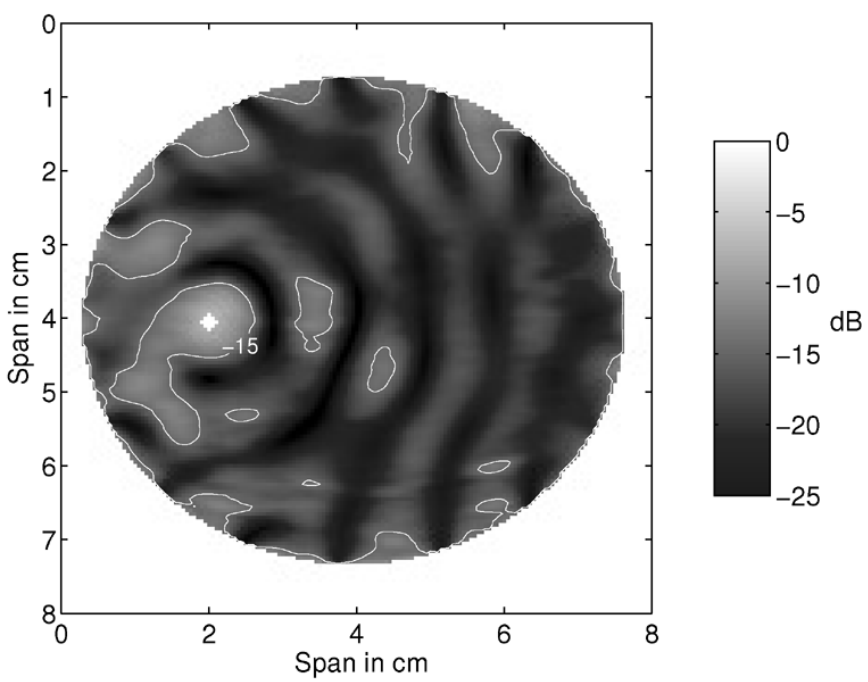

(b)

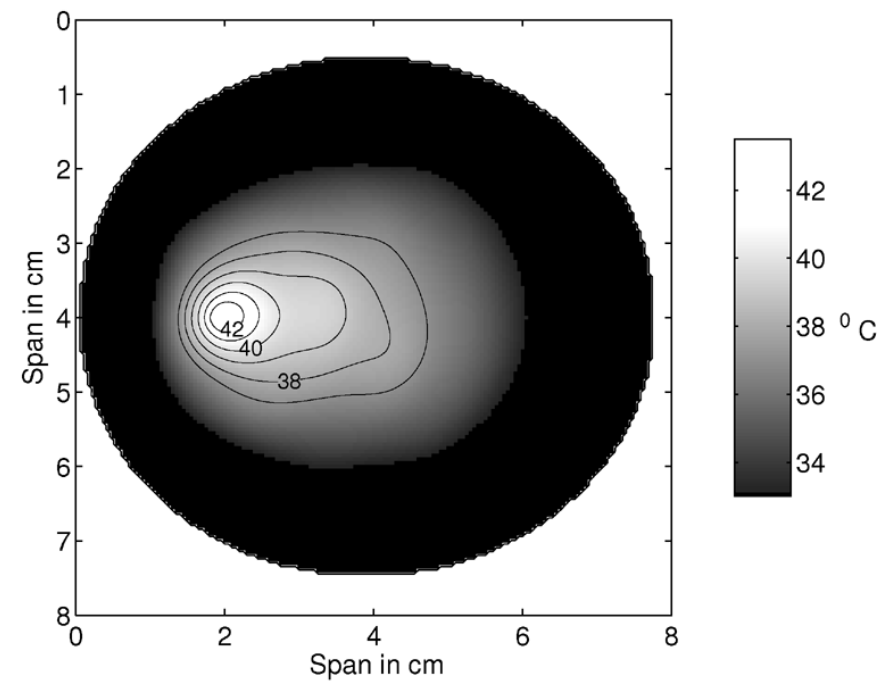

(d)

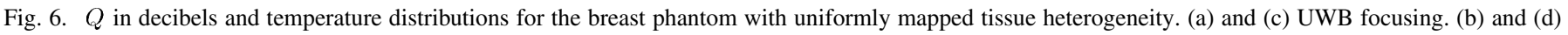
$\mathrm{NB}$ focusing at the optimal frequency of $3.5 \mathrm{GHz}$.

coordinates $(2.0,4.0) \mathrm{cm}$. We choose representative frequencies from the frequency band over which the UWB focusing signals have significant spectral content $(1-11 \mathrm{GHz})$. Fig. 4 shows the spatial distribution of $Q$ for the excitation frequencies of 2, 4, 6 , and $8 \mathrm{GHz}$. In each case, the maximum $Q$ occurs at the focus location, $\mathbf{r}_{f}=(2.0,4.0) \mathrm{cm}$. The presence of the tumor at this focus location introduces a local increase in the absorbed power density due to the higher conductivity of the tumor relative to the surrounding tissue. This selective absorption of the tumor augments the inherent focusing capability of the beamformer. Fig. 5 depicts the temperature profile that is obtained when each of the four $Q$ distributions of Fig. 4 are used as the input into the thermal model. Contour lines are shown in $1^{\circ} \mathrm{C}$ increments from $37^{\circ} \mathrm{C}$ to $42{ }^{\circ} \mathrm{C}$. The profiles of Fig. 5 show that the temperature gradients necessary to selectively heat the tumor site to therapeutic levels $\left(42^{\circ} \mathrm{C}-43^{\circ} \mathrm{C}\right)$ can be achieved at 2,4 , and (to some extent) $6 \mathrm{GHz}$, whereas, at $8 \mathrm{GHz}$, the temperature selectivity is severely degraded with temperatures reaching the highest levels near the surface of the breast instead of at the tumor site.
Several important trends can be observed in Figs. 4 and 5. Fig. 4 shows that an increase in frequency leads to higher resolution focusing, as was expected. In array processing terms, the width of the mainlobe of the transmit beampattern decreases with increasing frequency while the sidelobe levels increase. Fig. 4 also illustrates that an increase in excitation frequency leads to an increase in absorbed EM power near the surface of the breast. This is a direct consequence of attenuation increasing with frequency. A comparison between Figs. 4 and 5 reveals that, as the frequency is increased from 2 to $4 \mathrm{GHz}$, the EM power absorption at the tumor site becomes more localized, while the absorption near the surface does not increase significantly. As a result, the region surrounding the tumor that is exposed to temperatures above $42{ }^{\circ} \mathrm{C}$ is smaller at $4 \mathrm{GHz}$ relative to $2 \mathrm{GHz}$. As the frequency is increased further to $6 \mathrm{GHz}$, the EM power absorption near the surface increases considerably. Consequently, while the tumor site is still elevated to the desired temperature, the extent of the raised-temperature region away from the tumor site is enlarged relative to that observed at 


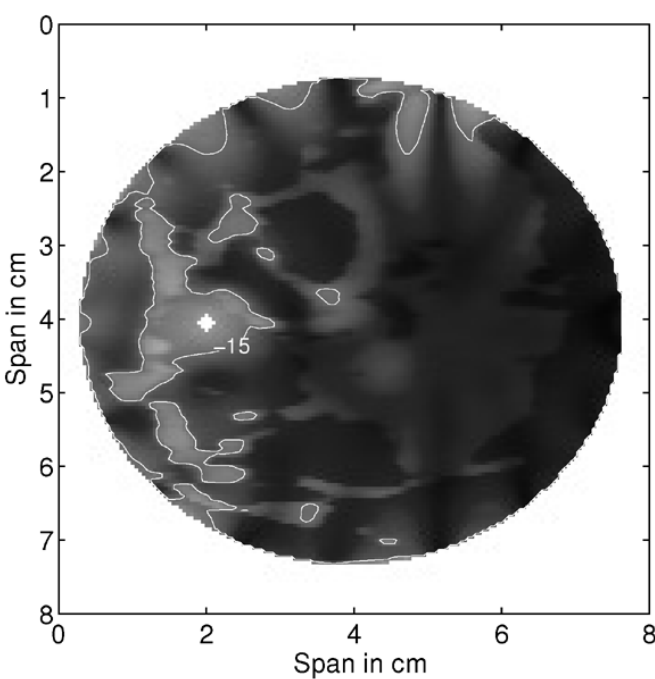

(a)

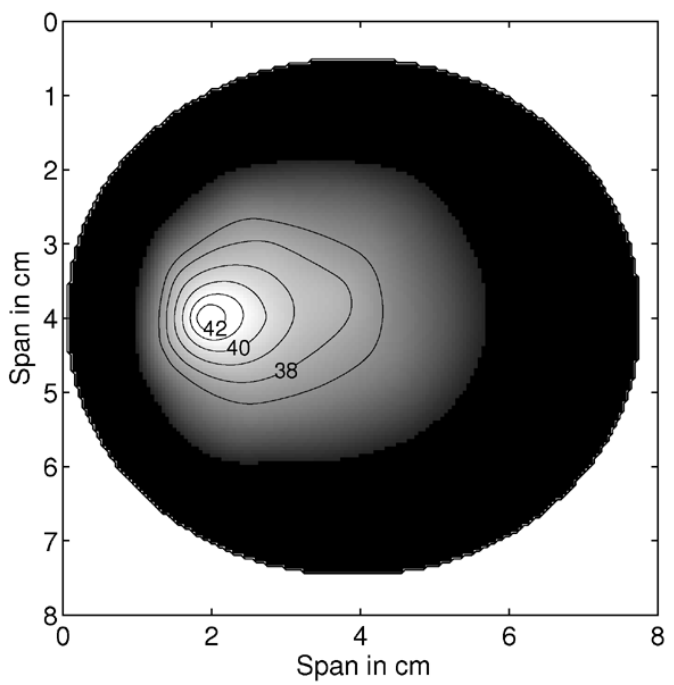

(c)
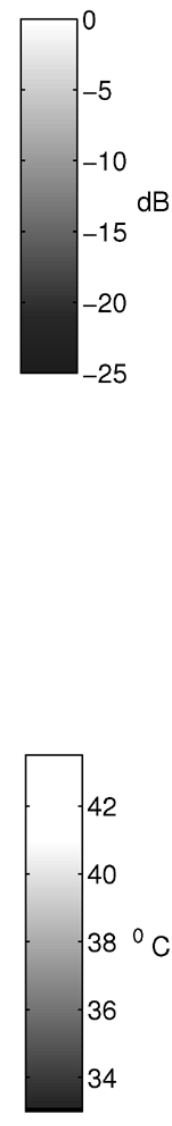

Fig. 7. $Q$ in decibels and temperature distributions for the breast phantom with piecewise linearly mapped tissue heterogeneity. (a) and (c) UWB focusing (b) and (d) NB focusing at the optimal frequency of $3.8 \mathrm{GHz}$. the lower frequencies. At $8 \mathrm{GHz}$, the increased sidelobe levels and the greatly increased EM power absorption in normal tissue result in ineffective hyperthermia treatment characterized by undesired hot spots near the surface of the breast.

Thus, a fundamental tradeoff exists when focusing NB signals in the breast. If too low of a frequency is chosen, then the extent of the region surrounding the tumor site that is raised to nonphysiological temperatures may be unnecessarily large [see Fig. 5(a)] due to poor focusing resolution associated with the mainlobe. If too high of a frequency is chosen, then too much of the transmitted power is absorbed in tissue near the breast surface and throughout the breast due to increased conductivity of normal tissue and higher sidelobe levels. This latter case results in auxiliary foci or hot spots in normal tissue rather than at the tumor site [see Fig. 5(d)]. Clearly, the choice of an effective NB excitation frequency requires balancing the competing demands of focusing resolution with depth of penetration within a propagation medium whose dielectric properties and volume are patient-specific.

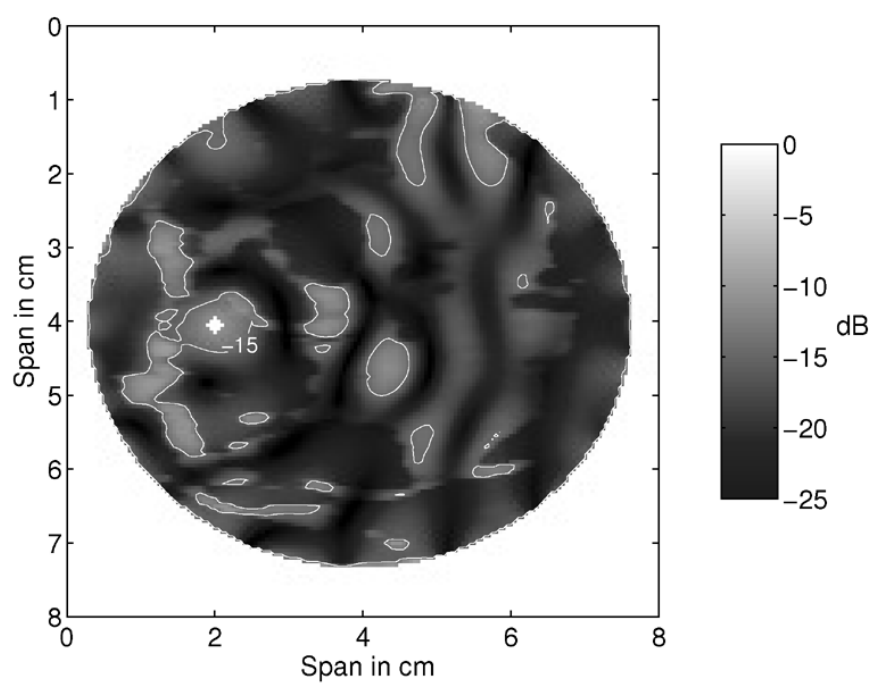

(b)

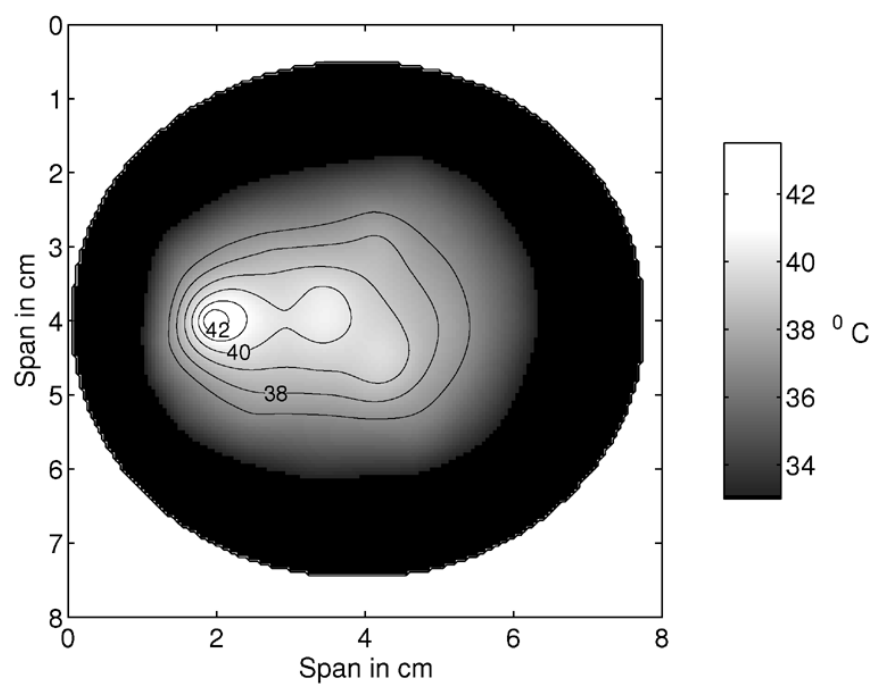

(d)
Although we only show results at four representative frequencies in the 1-11-GHz band, this frequency band can be further explored using finer sampling, and EM and thermal simulation can be performed at each frequency. The ratio of deposited power at the tumor location to deposited power near the breast surface can be calculated, and the frequency with the largest ratio is considered to be the "optimum" frequency. Note that the optimum frequency will vary with breast density and geometry. For the phantom with uniformly mapped tissue heterogeneity, the optimum frequency determined in this manner is $3.5 \mathrm{GHz}$. Interestingly, the spectral peak of transmit pulses that result from the UWB design described in Section II occurs at precisely this frequency.

The $Q$ and temperature profiles for the NB case at the optimum frequency compared with the UWB case are shown in Fig. 6. EM focusing in the vicinity of the tumor location appears to be comparable in both cases, leading to areas of similar size over which the temperature is elevated to above $42{ }^{\circ} \mathrm{C}$. In the normal tissue region in the center of the breast, the peak level 


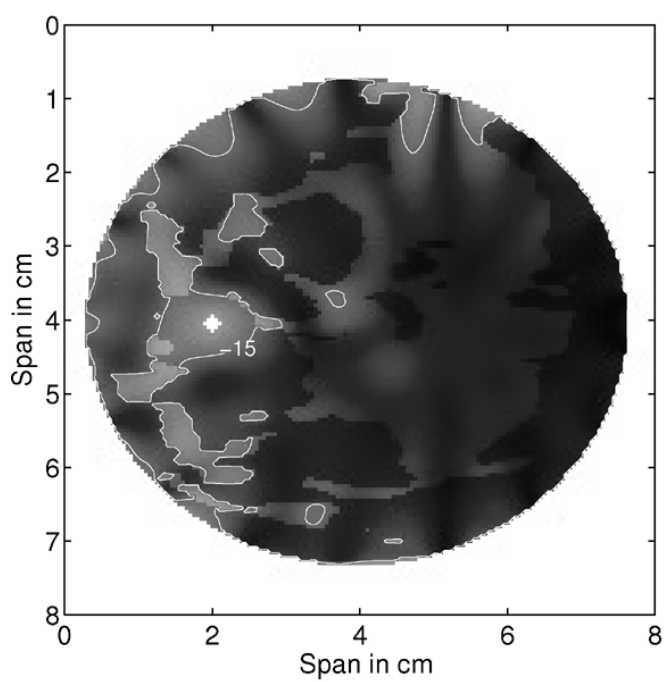

(a)

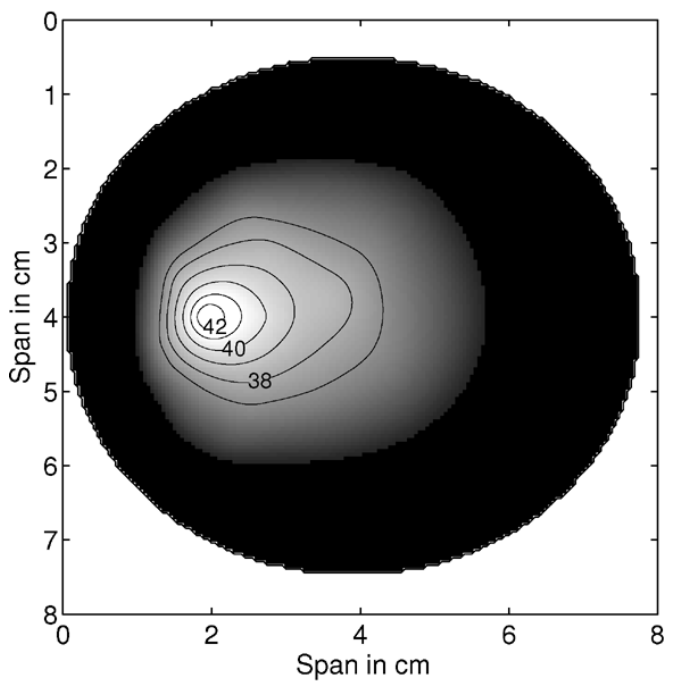

(c)
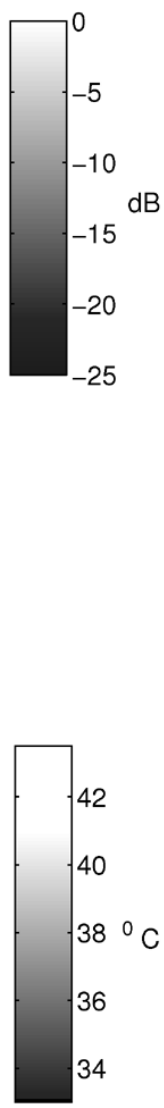

Fig. 8. $Q$ in decibels and temperature distributions for the breast phantom with bimodally mapped tissue heterogeneity. (a) and (c) UWB focusing. (b) and (d) $\mathrm{NB}$ focusing at the optimal frequency of $3.7 \mathrm{GHz}$. of absorbed EM power is $3 \mathrm{~dB}$ higher for the NB case. This extra absorbed power is the likely cause for the higher temperatures that appear around the center of the breast in the NB profile of Fig. 6(d) relative to the UWB profile of Fig. 6(c). Although UWB focusing yields higher absorbed power near the surface of the breast, the water cooling of the skin prevents hot spots from occurring in this region.

Fig. 7 shows the $Q$ and temperature profiles for UWB and NB focusing in the breast phantom with piecewise linearly mapped tissue heterogeneity. The optimal NB frequency here is $3.8 \mathrm{GHz}$. Increased power deposition can be seen throughout the breast in both Fig. 7(a) and (b) when compared with Fig. 6(a) and (b), although a greater increase occurs in the NB case. The increase is attributed to the sharper contrast in dielectric properties between fatty and fibroglandular tissue. A comparison between the UWB case in Fig. 7(a) and the NB case in Fig. 7(b) once again suggests that the excess power deposited near the center of the breast with NB focusing contributes to the degraded temperature profile in Fig. 7(d) compared with the temperature profile in Fig. 7(c).

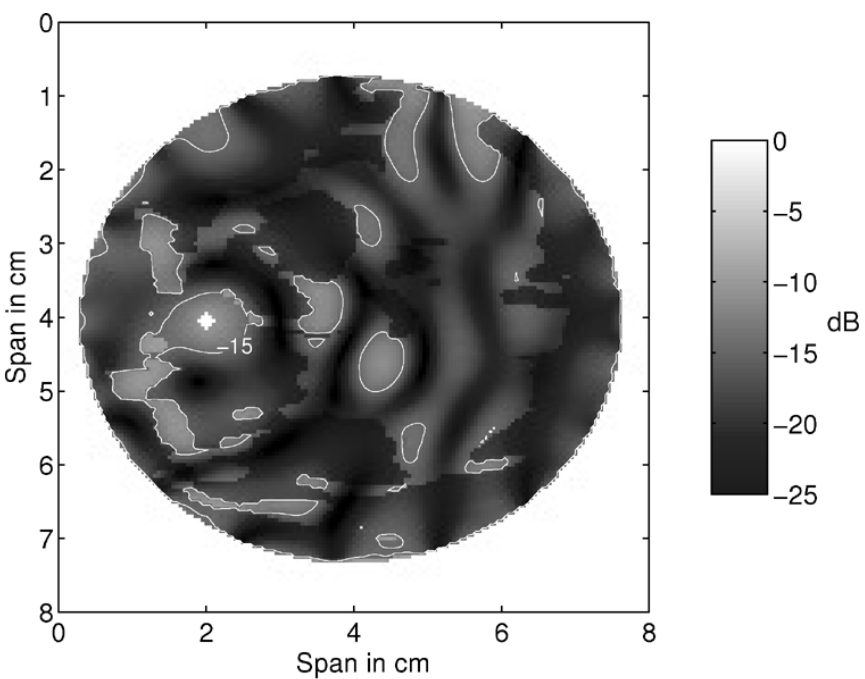

(b)
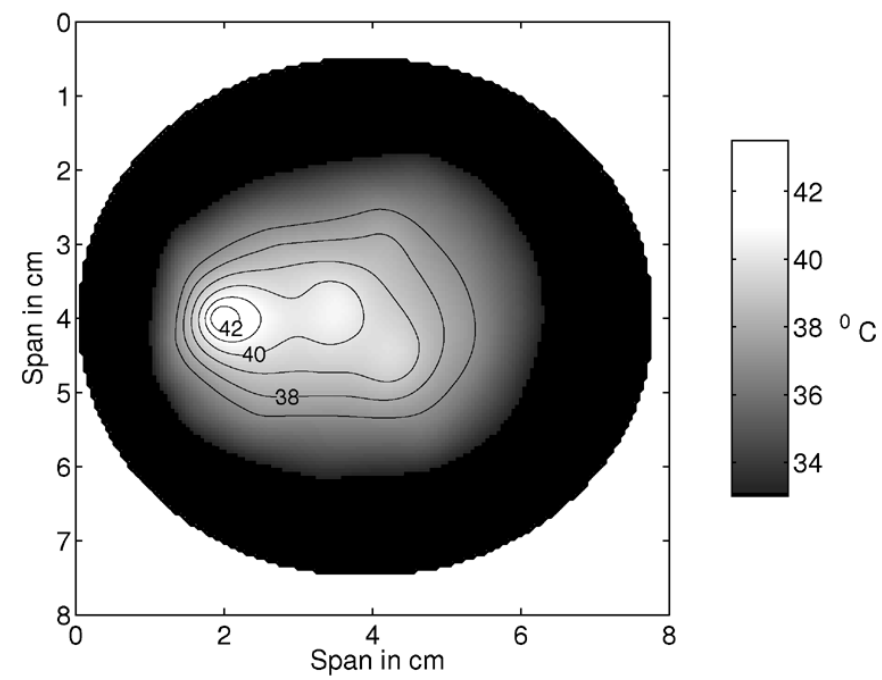

(d)
The resulting $Q$ and temperature profiles from NB and UWB focusing in the breast phantom with the bimodally mapped tissue heterogeneity is depicted in Fig. 8. The optimal NB frequency here is $3.7 \mathrm{GHz}$. Once more, UWB focusing yields a temperature profile with steeper gradients compared to the NB focusing profile. Figs. 6-8 consistently show that power absorption near the surface of the breast has no adverse effect on the temperature gradients, assuming that there is sufficient water cooling. The input power to the antennas for the UWB and optimal NB focusing solutions ranged from 4.5 to $9.5 \mathrm{~W} / \mathrm{cm}$.

\section{CONCLUSION}

In this study, we have compared UWB and NB beamforming for microwave hyperthermia treatment of breast cancer using anatomically realistic numerical breast phantoms containing a 2-mm-diameter tumor. Three methods for mapping MRI pixel intensity data to complex permittivity data were used to derive the phantoms. These mapping methods cover a wide range of possible embodiments of the dielectric-properties transitions 
that may occur in the breast interior due to heterogeneous tissue composition. The small tumor size creates one of the most challenging focusing scenarios for selective heating. This formal comparison study confirms our hypothesis that UWB focusing methods offer the potential for tighter focusing and a greater reduction of hot-spots compared to NB methods for a small $(<1$ $\mathrm{cm})$ tumor size. The results demonstrate that UWB focusing consistently produces the necessary temperature gradients required for effective hyperthermia treatment while preserving normal physiological temperatures throughout larger regions of normal tissue relative to NB focusing with an optimum excitation frequency, particularly in breast tissue environments that exhibit distinct dielectric-properties boundaries between fat and fibroglandular tissue.

\section{REFERENCES}

[1] K. A. Leopold, M. Dewhirst, T. Samulski, J. Harrelson, J. A. Tucker, S. L. George, R. K. Dodge, W. Grant, S. Clegg, L. R. Prosnitz, and J. R. Oleson, "Relationships among tumor temperature, treatment time, and histopathological outcome using preoperative hyperthermia with radiation in soft-tissue sarcomas," Int. J. Rad. Oncol. Biol. Phys., vol. 22, no. 5, pp. 989-998, 1992.

[2] J. van der Zee, D. G. Gonzalez, G. C. van Rhoon, J. D. P. van Dijk, W. L. J. van Putten, and A. A. M. Hart, "Comparison of radiotherapy alone with radiotherapy plus hyperthermia in locally advanced pelvic tumors: A prospective, randomised, multicentre trial," Lancet, vol. 355, pp. 1119-1125, 2000.

[3] P. Wust, B. Hildebrandt, G. Sreenivasa, B. Rau, J. Gellermann, H. Riess, R. Felix, and P. M. Schlag, "Hyperthermia in combined treatment of cancer," Lancet Oncol., vol. 3, no. 8, pp. 487-497, Aug. 2002.

[4] J. Overgaard, D. Gonzalez, M. Hulshof, G. Arcangeli, O. Dahl, O. Mella, and S. Bentzen, "Randomized trial of hyperthermia as an adjuvant to radiotherapy for recurrent or metastatic malignant melanoma," Lancet, vol. 345, pp. 540-543, 1995.

[5] C. C. Vernon, J. W. Hand, S. B. Field, D. Machin, J. B. Whaley, J. van der Zee, W. L. J. van Putten, G. C. van Rhoon, J. D. P. van Dijk, D. G. Gonzalez, F. F. Liu, P. Goodman, and M. Sherar, "Radiotherapy with or without hyperthermia in the treatment of superficial localized breast cancer: Results from five randomized controlled trials," Int. J. Rad. Oncol. Biol. Phys., vol. 35, no. 4, pp. 731-744, Jul. 1996.

[6] J. W. Hand, D. Machin, C. C. Vernon, and J. B. Whaley, "Analysis of thermal parameters obtained during phase III trials of hyperthermia as an adjunct to radiotherapy in the treatment of breast carcinoma," Int. J. Hyperthermia, vol. 13, pp. 343-364, 1997.

[7] D. S. Kapp, "Efficacy of adjuvant hyperthermia in the treatment of superficial recurrent breast cancer: Confirmation and future directions," Int. J. Rad. Oncol. Biol. Phys., vol. 35, pp. 1117-1121, 1996.

[8] M. E. Kowalski, B. Behnia, A. G. Webb, and J.-M. Jin, "Optimization of electromagnetic phased-arrays for hyperthermia via magnetic resonance temperature estimation," IEEE Trans. Biomed. Eng., vol. 49, no. 11, pp. 1229-1241, Nov. 2002.

[9] A. J. Fenn, V. Sathiaseelan, G. A. King, and P. R. Stauffer, "Improved localization of energy deposition in adaptive phase-array hyperthermia treatment of cancer," Linc. Lab. J., vol. 9, no. 2, pp. 187-195, 1996.

[10] S. Jacobsen, "Reduction of hot spots in hyperthermia by means of broadband energy transmission," Electron. Lett., vol. 34, no. 20, pp. 1901-1902, Oct. 1998.

[11] M. C. Converse, E. J. Bond, S. C. Hagness, and B. D. Van Veen, "Ultrawide-band microwave space-time beamforming for hyperthermia treatment of breast cancer: A computational feasibility study," IEEE Trans. Microw. Theory Tech., vol. 52, no. 8, pp. 1876-1889, Aug. 2004.

[12] H. Cox, R. M. Zeskind, and M. M. Owen, "Robust adaptive beamforming," IEEE Trans. Acoust., Speech Signal Process., vol. ASSP-35, pp. $1365-1375$, Oct. 1987.

[13] A. Taflove and S. C. Hagness, Computational Electrodynamics: The Finite-Difference Time-Domain Method, 3rd ed. Boston, MA: Artech House, 2005.

[14] E. J. Bond, X. Li, S. C. Hagness, and B. D. Van Veen, "Microwave imaging via space-time beamforming for early detection of breast cancer," IEEE Trans. Antennas Propag., vol. 51, no. 8, pp. 1690-1705, Aug. 2003.
[15] J. Z. Bao, M. L. Swicord, and C. C. Davis, "Microwave dielectric characterization of binary mixtures of water, methanol, and ethanol," $J$. Chem. Phys., vol. 104, no. 12, pp. 4441-4450, Mar. 1996.

[16] S. Gabriel, R. W. Lau, and C. Gabriel, "The dielectric properties of biological tissues: III. Parametric models for the dielectric spectrum of tissues," Phys. Med. Biol., vol. 41, pp. 2271-2293, 1996.

[17] W. D. Hurt, J. M. Ziriax, and P. A. Mason, "Variability in EMF permittivity values: Implications for SAR calculations," IEEE Trans. Biomed. Eng., vol. BME-27, no. 10, pp. 396-401, Oct. 1987.

[18] S. S. Chaudhary, R. K. Mishra, A. Swarup, and J. M. Thomas, "Dielectric properties of normal and malignant human breast tissues at radiowave and microwave frequencies," Indian J. Biochem. Biophys., vol. 21, pp. 76-79, 1984.

[19] A. J. Surowiec, S. S. Stuchley, J. R. Barr, and A. Swarup, "Dielectric properties of breast carcinoma and the surrounding tissues," IEEE Trans. Biomed. Eng., vol. 35, no. 4, pp. 257-263, Apr. 1988.

[20] W. T. Joines, Y. Zhang, C. Li, and R. L. Jirtle, "The measured electrical properties of normal and malignant human tissues from 50 to 900 MHz," Med. Phys. J., vol. 21, no. 4, pp. 547-550, 1994.

[21] P. M. Meaney, M. W. Fanning, D. Li, S. P. Poplack, and K. D. Paulsen, "A clinical prototype for active microwave imaging of the breast," IEEE Trans. Microw. Theory Tech., vol. 48, no. 11, pp. 1841-1853, Nov. 2000.

[22] A. M. Campbell and D. V. Land, "Dielectric properties of female human breast tissue measured in vitro at $3.2 \mathrm{GHz}$," Phys. Med. Biol., vol. 37, no. 1, pp. 193-210, 1992.

[23] C. Beasley, S. C. Hagness, J. Booske, T. M. Breslin, F. Xu, M. J. Lindstrom, D. Popovic, M. Okoniewski, W. Temple, A. Magliocco, and T. Ogilvie, "Ex-vivo dielectric characterization of normal, benign, and malignant breast tissue at microwave frequencies: Preliminary results from a multi-institutional study," presented at the IEEE Int. Symp. Antennas Propag. USNC/URSI Radio Sci. Meeting, Columbus, OH, Jun. 2003.

[24] X. Li and S. C. Hagness, "A confocal microwave imaging algorithm for breast cancer detection," IEEE Microw. Wireless Compon. Lett., vol. 11, no. 3, pp. 130-132, Mar. 2001.

[25] O. P. Gandhi and C. M. Furse, "Currents induced in the human body for exposure to ultrawideband electromagnetic pulses," IEEE Trans. Electromagn. Compat., vol. 39, no. 2, pp. 174-180, 1997.

[26] P. Bernardi, M. Cavagnaro, S. Pisa, and E. Piuzzi, "Specific absorption rate and temperature elevation in a subject exposed in the far-field of radio-frequency sources operating in the $10-900-\mathrm{MHz}$ range," IEEE Trans. Biomed. Eng., vol. 50, no. 3, pp. 295-304, Mar. 2003.

[27] K. Giering, I. Lamprecht, and O. Minet, "Specific heat capacities of human and animal tissues," Proc. SPIE, vol. 2624, pp. 188-197, Jan. 1996.

[28] H. Bowmann, "Heat transfer and thermal dosimetry," J. Microw. Power E.E., vol. 16, no. 2, pp. 121-130, 1981.

[29] K. Sekins and A. Emery, "Thermal modeling of local hyperthermia," in Hyperthermia in Cancer Treatment. Boca Raton, FL: CRC, 1986, vol. 2 , ch. 6.

[30] M. P. Robinson, M. J. Richardson, J. L. Green, and A. W. Preece, "New materials for dielectric simulation of tissues," Phys. Med. Biol., vol. 36, no. 12, pp. 1565-1571, Dec. 1991.

[31] K. R. Diller, J. W. Valvano, and J. A. Pearce, "Bioheat transfer," in The CRC Handbook of Thermal Engineering. Boca Raton, FL: CRC, 2000.

[32] J. Lang, B. Erdmann, and M. Seebass, "Impact of nonlinear heat transfer on temperature control in regional hyperthermia," IEEE Trans. Bio. Eng., vol. 46, no. 9, pp. 1129-1138, Sep. 1999.

[33] H. L. M. Cheng and D. B. Plewes, "Tissue thermal conductivity by magnetic resonance thermometry and focused ultrasound heating," $J$. Mag. Res. Imag., vol. 16, no. 5, pp. 598-609, Nov. 2002.

[34] S. J. Graham and M. J. Bronskill, "MR measurement of relative water content and multicomponent $T_{2}$ relaxation in human breast," Mag. Res. Med., vol. 35, no. 5, pp. 706-715, May 1996.

[35] J. S. Al-bahri and N. M. Spyrou, "Photon linear attenuation coefficients and water content of normal and pathological breast tissues," Appl. Rad. Iso., vol. 47, no. 8, pp. 777-784, Aug. 1996.

[36] H. Q. Woodard and D. R. White, "The composition of body tissues," Brit. J. Rad., vol. 59, pp. 1209-1219, Dec. 1986.

[37] S. J. Graham, P. L. Stanchev, J. O. A. LloydSmith, M. J. Bronskill, and D. B. Plewes, "Changes in fibroglandular volume and water content of breast tissue during the menstrual cycle observed by MR imaging at 1.5 T," J. Mag. Res. Imag., vol. 5, no. 6, pp. 695-701, Nov. 1995 . 
[38] J. Werner and M. Buse, "Temperature profiles with respect to inhomogeneity and geometry of the human body," J. Appl. Physiol., vol. 65, no. 3, pp. 1110-1118, Sep. 1988.

[39] F. A. Duck, Physical Properties of Tissue: A Comprehensive Reference Book. London, U.K.: Academic, 1990.

[40] E. Y. K. Ng and N. M. Sudharsan, "Effect of blood flow, tumour and cold stress in a female breast: A novel time-accurate computer simulation," Proc. Int. Mech. Eng. Part H-J. Eng. Med., vol. 215, no. H4, pp. 393-404, 2001.

[41] B. I. Tropea, "Thermal injury kinetic in electrical trauma," Trans. ASME, J. Biomed. Eng., vol. 114, no. 21, pp. 4096-4105, Jul. 1992.

[42] J. C. Chato, "Fundamentals of bioheat transfer," in Thermal Dosimetry and Treatment Planning. Berlin, Germany: Springer-Verlag, 1990, pp. $1-56$.

[43] A. Shitzer and R. C. Eberhart, Heat Transfer in Medicine and Biology: Analysis and Applications. New York, NY: Plenum, 1985.

[44] N. M. Fried, Y. D. Sinelnikov, B. B. Pant, W. W. Roberts, and S. B. Solomon, "Noninvasive vasectomy using a focused ultrasound clip: Thermal measurements and simulations," IEEE Trans. Biomed. Eng., vol. 48, no. 12, pp. 1453-1459, Dec 2001.

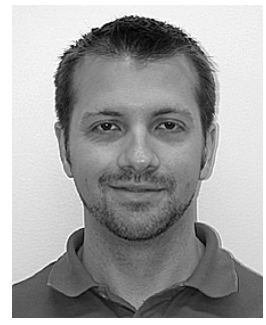

Mark Converse (S'92-M'04) received the B.S. degree in electrical engineering and the M.S. and Ph.D. degrees from the University of Wisconsin-Madison, in 1996, 1999, and 2003, respectively.

During his graduate studies, he was engaged in plasma processing research involving damage evaluation/analysis and mitigation during the etching process. After receiving the M.S. degree in 1999, he began research in microwave vacuum electronics, investigating the impulse response of the helix traveling wave tube. In May of 2003, he began postdoctoral research examining the feasibility of using ultra-wideband microwave hyperthermia to treat breast cancer. Currently, he is an Assistant Scientist with the University of Wisconsin-Madison, where he is investigating microwave ablation of liver cancer. His research interests include EM interactions with materials, electrical/biological interfaces, and organic electronics.

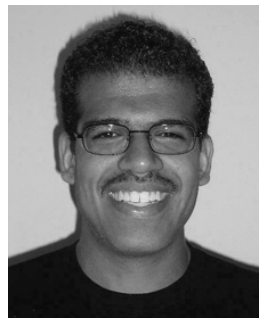

Essex J. Bond (S'98-M'06) received the B.S. degree from the Milwaukee School of Engineering, Milwaukee, WI, in 1999, the M.S. degree from the University of Wisconsin-Madison, in 2001, both in electrical engineering, and is currently working toward the Ph.D. degree in electrical and computer engineering at the University of Wisconsin-Madison.

His research interests include biomedical applications of signal processing and array signal processing.

Mr. Bond is the recipient of a National Institutes of Health Pre-doctoral Fellowship.

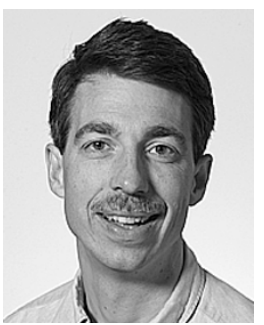

Barry D. Van Veen (S'81-M'86-SM'97-F'02) was born in Green Bay, WI. He received the B.S. degree from Michigan Technological University, Houghton, in 1983, and the Ph.D. degree from the University of Colorado at Boulder, in 1986, both in electrical engineering.

In the spring of 1987, he was with the Department of Electrical and Computer Engineering, University of Colorado at Boulder. Since August of 1987, he has been with the Department of Electrical and Computer Engineering, University of Wisconsin-Madison, where he is currently a Professor. His research interests include signal processing for sensor arrays and biomedical applications of signal processing. He coauthored Signals and Systems (Wiley, 1999, 1st ed., 2003, 2nd ed.).

Dr. Van Veen served as an Associate Editor for the IEEE TRANSACTIONS ON SignAl Processing and on the IEEE Signal Processing Society's Statistical Signal and Array Processing Technical Committee and the Sensor Array and Multichannel Technical Committee. He was a recipient of a 1989 Presidential Young Investigator Award from the National Science Foundation and a 1990 IEEE Signal Processing Society Paper Award. He was also the recipient of the Holdridge Teaching Excellence Award from the Electrical and Computer Engineering Department at the University of Wisconsin in 1997. He was an Office of Naval Research Fellow while working on the Ph.D. degree.

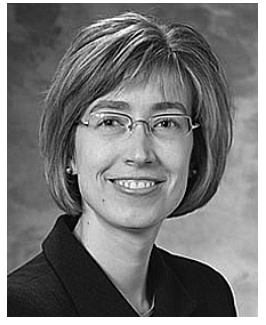

Susan C. Hagness (S'91-M'98-SM'04) received the B.S. degree (with highest honors) and Ph.D. degree in electrical engineering from Northwestern University, Evanston, IL, in 1993 and 1998, respectively.

Since August 1998, she has been with the University of Wisconsin-Madison, where she is currently an Associate Professor with the Department of Electrical and Computer Engineering and a faculty affiliate of the Department of Biomedical Engineering. Her bioelectromagnetics research interests include microwave imaging, sensing, and thermal therapy techniques, UWB radar, dielectric spectroscopy, time-domain inverse scattering, and FDTD theory and applications in biology and medicine. She coauthored Computational Electrodynamics: The Finite-Difference Time-Domain Method (Artech House, 2005, 3rd ed.)

Dr. Hagness served as an elected member of the IEEE Antennas and Propagation Society (AP-S) Administrative Committee from 2003 to 2005. She is currently serving as a member-at-large of the United States National Committee (USNC) of the International Union of Radio Science (URSI) and as an Associate Editor for the IEEE ANTENNAS AND WIRELESS PROPAGATION LETTERS. She was the recipient of the Presidential Early Career Award for Scientists and Engineers presented by the White House in 2000. In 2002, she was named one of the 100 top young innovators in science and engineering in the world by the Massachusetts Institute of Technology (MIT) Technology Review magazine. She was the recipient of the University of Wisconsin Emil Steiger Distinguished Teaching Award in 2003, the IEEE Engineering in Medicine and Biology Society Early Career Achievement Award in 2004, and the URSI Isaac Koga Gold Medal in 2005. While working toward the Ph.D. degree, she was a National Science Foundation Graduate Fellow and a Tau Beta Pi Spencer Fellow. 